\title{
Reviewer Acknowledgements for Global Journal of Health Science, Vol. 9, No. 1
}

Global Journal of Health Science wishes to acknowledge the following individuals for their assistance with peer review of manuscripts for this issue. Their help and contributions in maintaining the quality of the journal are greatly appreciated.

Global Journal of Health Science is recruiting reviewers for the journal. If you are interested in becoming a reviewer, we welcome you to join us. Please find the application form and details at http://recruitment.ccsenet.org and e-mail the completed application form to gjhs@ccsenet.org.

\section{Reviewers for Volume 9, Number 1}

\begin{tabular}{|c|c|}
\hline Abdul Haseeb & Kartheek R. Balapala \\
\hline Abdul Raziq & Kristopher Day \\
\hline Abdullah Mohammed Alzahem & Leena Kunnath Chacko \\
\hline Abin Varghese & Liu Liu \\
\hline Ama Fenny & Luba Ivanov \\
\hline Angus Nnamdi Oli & Maja Racic \\
\hline Ashwini Kucknoor & Melissa Freizinger \\
\hline Bashiru Saeed & Meng Zhao \\
\hline Christy $\mathrm{Pu}$ & Montarat Thavorncharoensap \\
\hline Chung-Yu Chen & Mustafa Kaplan \\
\hline Conceição Martins & Natasha Azzopardi Muscat \\
\hline David Walwyn & Nima Hafezi Nejad \\
\hline Deborah A. Saber & Nishith H Vayada \\
\hline Delfina Ramos & Piacherski Valery \\
\hline Dilek Pirim & Piacherski Valery \\
\hline Ekta Singh Dahiya & Pi-Ming Yeh \\
\hline Esteban Ortiz-Prado & Polly Yeung \\
\hline Francisco Rodenas Rigla & Raymond Jagessar \\
\hline Gabriele Messina & Reşat \\
\hline Georgann Weissman & Rhonda Brown \\
\hline Hadii M Mamudu & Sara Melo \\
\hline Heba Aboel Naga & Shivank Madan \\
\hline Helen Smits & Shozab Ali \\
\hline Hongfeng Jiang & Snehal Padhye \\
\hline Isac Silva De Jesus & Suleyman Gorpelioglu \\
\hline Jan Chrastina & Tan Ching Siang \\
\hline Jan Florin & Thomas Wolfgang Leiblein \\
\hline Joanne Cleary-Holdforth & Trisha Dunning \\
\hline Jose Hernandez-Padilla & Tsan Yang \\
\hline José Joaquín Mira & Xiaosun Lu \\
\hline Jose R. Cordon & Yuan Zhang \\
\hline Karen Chan & \\
\hline Kartalis Nikos & \\
\hline
\end{tabular}

